\title{
ILLUSTRATE WELL TO GET NOTICED: GRAPHS AND FIGURES IN RESEARCH PAPERS
}

\author{
Samir Kumar Praharaj \\ Professor, Department of Psychiatry, Kasturba Medical College, Manipal, Karnataka. \\ Correspondence: Department of Psychiatry, Kasturba Medical College, Manipal, Karnataka. E-mail: samirpsyche@yahoo.co.in
}

\section{INTRODUCTION}

Figures (and tables) are important ways to present data succinctly. If done properly, they not only reduce word count but also convey an important message in a meaningful way so that the readers can grasp it easily. Figures include graphs, photographs, sketches, flow charts, etc. Figures can be used to describe data in unique ways which may be difficult to put in words. As the saying goes, "a picture is worth a thousand words." Essentially, figures communicate an important point at a glance. Line graphs show trends, pie charts show relative proportions of a whole, and photographs provide proof of findings. If the data do not show any specific trend or relationship, then a graph should not be used.

The major types of figure artwork include line art and continuous-tone art. Line arts includes black markings on a white background, i.e. lines, solid shapes, and letters, as in line graphs, scatter plots, flowcharts, etc. Continuous-tone arts includes colors or shades of grey (e.g. photographs, sketches). Sometimes, grey-scale line arts are used in which different shades of grey are used in bar charts, pie charts, etc. to distinguish different sections.

\section{GENERAL GUIDELINES}

The illustration should be meaningful by itself, and the reader should not have to refer the text to comprehend a graph or figure. The matter provided in the illustrations should not be repeated in the text, and vice versa. However, one should link the illustrations to the text and can highlight the main finding.

The number of figures to be used in a manuscript depends on the number of key trends that need to be highlighted. However, too many figures can disrupt the reader's focus. The number of figures that are permitted for a particular kind of manuscript is usually specified in the instructions to the authors.

Choosing the correct graph is the most important step. It is not uncommon to see lines connecting discrete data. Bar graphs are suited for categorical data, whereas box plots are better for continuous data. If both dependent and independent variable are continuous, a scatterplot is the best option to depict such data.

Color figures are costly, and most journals charge additionally for them (though web version of manuscripts can have color figures without additional charge). Most figures and photographs can be prepared in black and white - Only rarely does a color figure add something extra to the reader's understanding.

In some situations, actual photographs are invaluable. Examples include photos of patient's body parts and images from pathology and neuroimaging. In general, the photographs should

Please cite this article as Praharaj SK. Illustrate well to get noticed: Graphs and figures in research papers. Kerala Journal of Psychiatry 2017; 30(2):115-9. DOI: 10.30834/KJP.30.2.2018.135 
be clear and of high quality so that they are not blurred or distorted with size reduction that can happen during the publication process.

If photographs are used, patient confidentiality is of utmost importance. One way to reduce possible identification of subjects is to mask both the eyes with a black rectangular area. Sometimes, blurring that area using software can have a similar effect. If it is not possible to hide the details (e.g. when eye signs are important), it is imperative to obtain written informed consent, and this can be mentioned in the figure legend.

Previously, figures used to be submitted as cameraready artworks. Nowadays most figures and photographs are prepared, stored and submitted in electronic format. The files submitted electronically should have higher resolution, i.e. higher pixels per inch (ppi) or dots per inch (dpi).

\section{PRINCIPLES FOR MAKING FIGURES}

The various elements of a graph include:

1. Figure number,

2. Caption,

3. Data field,

4. Axes and scales,

5. Symbols, and

6. Source line (All the components are not necessarily present in every graph).

Most graphs are prepared using statistical programs. There are certain basic principles that must be adhered to while preparing various elements of graphs:

Figure number: The figures are numbered sequentially in the order in which they appear in the text (Figure 1, Figure 2, etc.). The parts of a figure may be designated in a consistent way (e.g. Figure 1a, Figure 1b or Figure 1A, Figure 1B). All the figures should be cited in the text (e.g. Figure 1 shows... or ... as shown in Figure 1).

Caption (Title, Legend): All figures should have a figure number followed by a brief, informative description. Usually, it is not in a sentence format (e.g. Figure 1: Mean changes in HAM-D scores over time). If needed, it can be longer and use standard punctuation marks. The caption should be understandable without referring to the text.

Axes: Usually, the independent variable is drawn in the horizontal x-axis (abscissa) and dependent variable in the vertical $y$-axis (ordinate). Therefore, time is always drawn on the $x$-axis. The length of the two axes are balanced (approximately 1:1 ratio).

Data points and scale: The scale used should match the range of data. The $\mathrm{x}$-axis and the $\mathrm{y}$-axis should not be extended much beyond the data points, as it would result in large empty spaces in the graph (e.g. If data points range from 0 to 23 , maximum index number can be 25, not 50). Also, truncating, enlarging and compressing data points can be misleading for the readers (e.g. for data points ranging from 23 to 29, indexing from 20 to 30, rather than 0 to 30). Extending a regression line beyond the actual data points is also discouraged. Tick marks are usually placed outside the axis line (not through or inside).

Symbols: Use standard, well-accepted symbols for data points. The symbols should be distinct and provide good contrast from the background. Common ones include closed or open circles, triangles, squares, or diamonds that can be used in different combinations. For example, to represent two sets of data, open circles and closed squares can be used. The symbols should be at least $2 \mathrm{~mm}$ in size.

Source line: To reproduce any figure or photograph that has been published elsewhere, written permission is required from the publishers (i.e. copyright owners). It can be mentioned as: 
Figure 1a: Frequency distribution of types of alcohol use (3D graph)

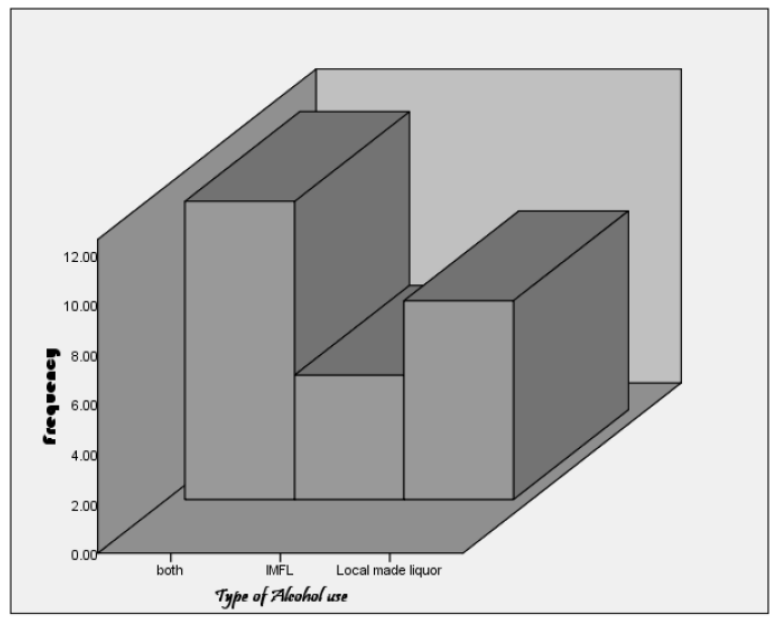

Reprinted with permission from reference $\mathrm{xx}$. Copyright 2018 Indian Psychiatric Society.

If only a part of a figure is used, "reprinted" is replaced with "adapted."

Adapted with permission from Praharaj (2018). Copyright Year Copyright Owner's Name.

Clarity: This is the most important aspect of any graph. All graphs should be clear, without any ambiguity, and easily readable. The data points and lines should be easy to read and distinct. For lines, the minimum and maximum recommended widths are 0.5 points and 1.5 points respectively. Fonts that are plain and simple, such as Helvetica or Times Roman, are the best for scientific art.

Color: The background of the graphs should be white, and the graph elements should be in black. Symbols or shading can be used to depict different data sets. Print publication of color graphs are extremely costly and is avoided if the same information can be presented with black and white graphs.

Image quality: The graphs should be prepared with a higher resolution (i.e. $600 \mathrm{dpi}$ or higher).
Figure 1b: Frequency distribution of types of alcohol use (2D graph)

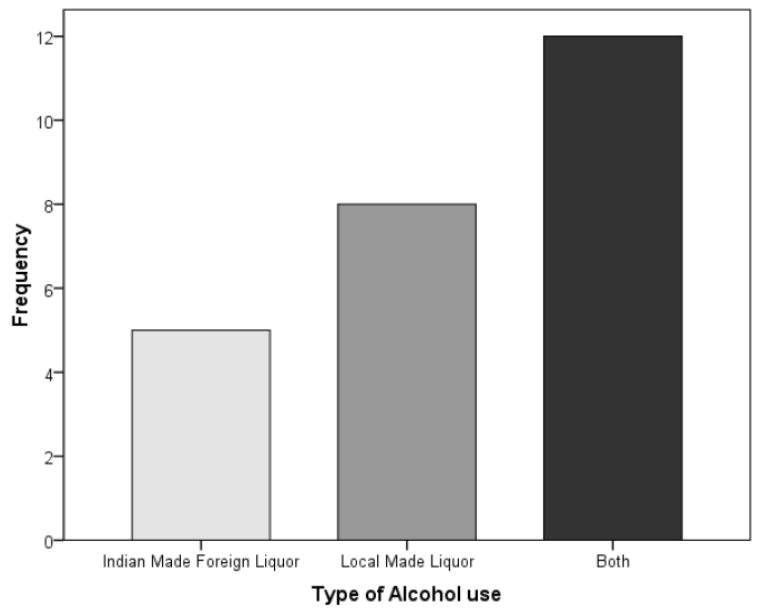

Those with a lower resolution would appear distorted in print. Photographs should be scanned at a minimum resolution of $300 \mathrm{dpi}$.

\section{ELECTRONIC FILE FORMATS}

Many software to draw, scan, and manipulate figures is commercially available. When the figure is saved in a format that can be opened only using that software, it is called an application file (e.g. Adobe Photoshop produces .psd files, CorelDraw produces .cdr files). Such file formats are better avoided as the publisher may not be using that software. There are certain file formats that are opened in many different software packages. For print, Encapsulated PostScript (EPS) or Tagged Image File Format (TIFF) are the preferred options. The quality of a TIFF image depends on the resolution, i.e. ppi or dpi. Graphics Interchange Format (GIF) is a pixel-based format similar to TIFF, but the resolution is preset at $72 \mathrm{ppi}$, which is appropriate for the Web versions but not sufficient for print. Joint Photographic Experts Group (JPEG) is another pixel-based format like TIFF and can be prepared with higher resolutions than GIFs. However, JPEGs are actually compressed files which lose small amounts of digital information every time they are opened, recompressed, and saved. Therefore, JPEG images tend to degrade 
Figure 2a: A verage test score in a group of persons

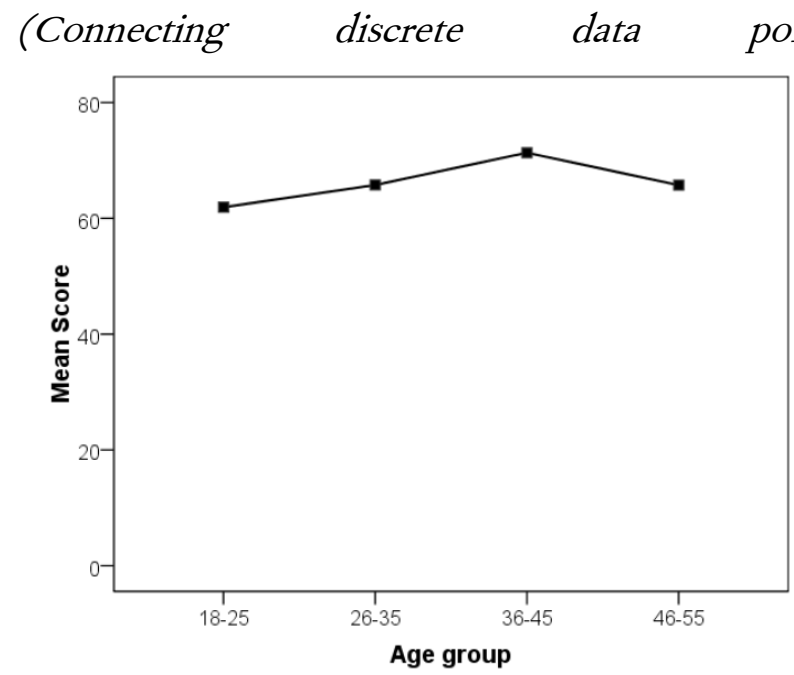

with repeated processing - Only "freshly prepared”, high-quality JPEGs are suitable for print publication.

\section{COMMON ERRORS WHILE MAKING GRAPHS}

Many statistical software produces graphs readily with several design features. However, many visually appealing, flashy graphs can be confusing and misleading. Three-dimensional graphs with skewed perspectives would be difficult to follow (see Figure 1a). Authors often forget that the font type, font size, number format, and background can be edited in the graphs produced in statistical software - Figure lb shows the same results in an improved format: the data points are not distorted in a skewed perspective, the category labels and number formats are more efficiently represented, the background is white, and the groups have different shades that allow easy differentiation. Graphs produced by computers can contain other errors, e.g. joining discrete data points as shown in Figure 2a (average test score in a group of persons). Connecting these points means that there are values between the age groups, which is not based on any observation. A correct way to display separate values is a bar chart in which the
Figure 2b: A verage test score in a group of persons (Bargraph)

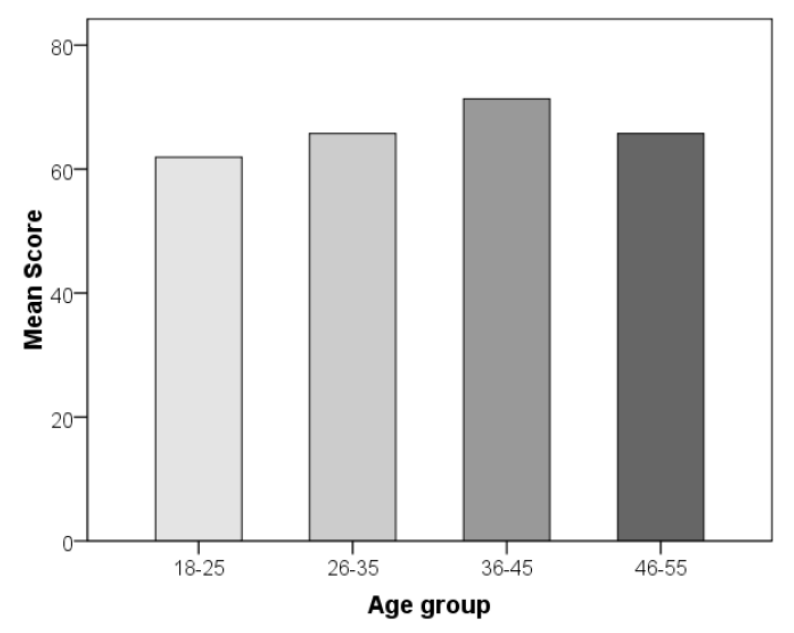

columns represent the average scores in each age group.

Figure 3a shows a change in mean scores in active and control groups, with a narrow scale range, giving a false impression of large change in scores. Such graphs can mislead the readers. Figure $3 \mathrm{~b}$ is a correct way of representing the facts, with score starting from zero; however, the lines are overlapping and may be difficult to differentiate. Figure $3 c$ is the best possible way to represent the facts without creating any false impressions.

\section{BEFORE YOU SUBMIT}

Submit the figures at the end of the manuscript, after the references, each of them in a separate page. Label each figure and ensure that they are linked to the text. The illustrations should be "inserted" (and not "pasted") into the text document so that they maintain their original resolution.

Some journals require the figures to be submitted as separate files; in such situations, the figure legends are mentioned in the manuscript file. Name the electronic files briefly (e.g. Praharaj_fig01.tiff). In the end, never forget to check how the figures and photographs appear in the proof copy of the manuscript. Maybe that would be your last chance to correct any error before the publication. 
Figure 3a: Changes in mean scores over time with narrow scale range

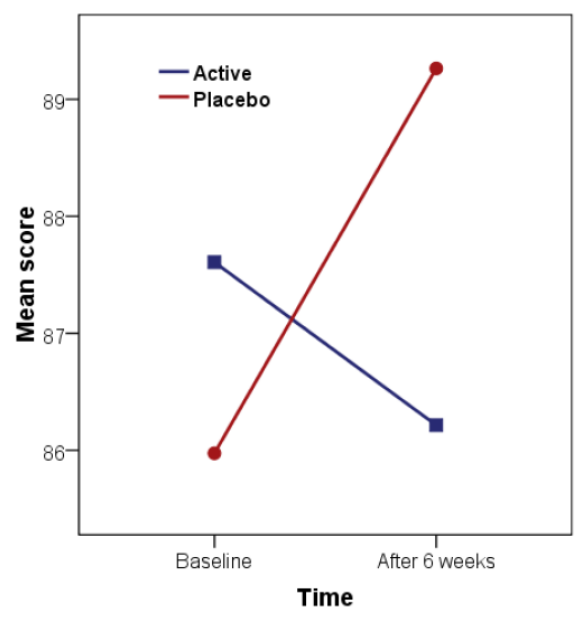

Figure 3c: Changes in mean scores over time with optimum scale range

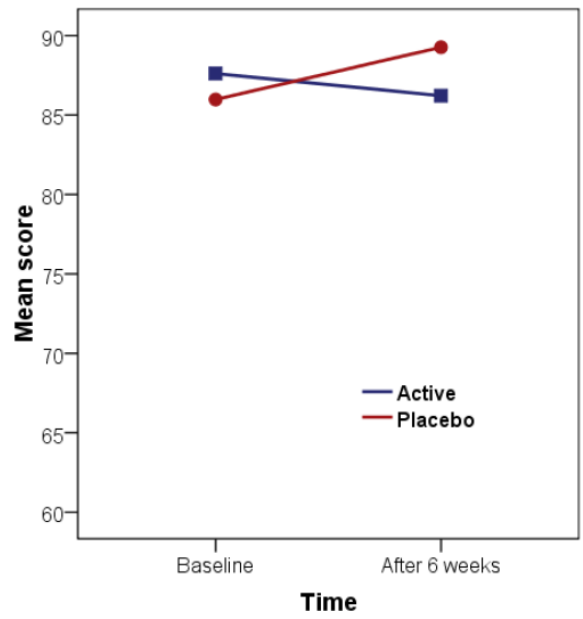

Figure 3b: Changes in mean scores over time with scale starting from zero

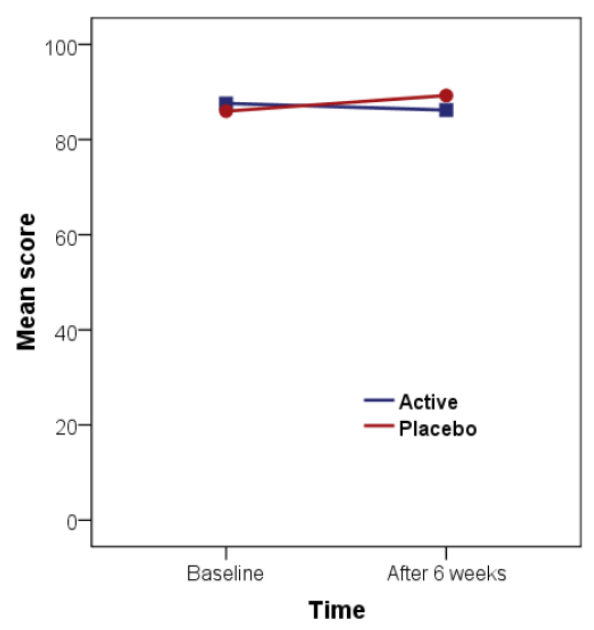

\section{SUGGESTED READING}

1. Coghill AM, Garson LR. The ACS Style Guide. Effective Communication of Scientific Information. 3rd ed. New York: Oxford University Press; 2006.

2. Franzblau LE, Chung KC. Graphs, tables, and figures in scientific publications: the good, the bad, and how not to be the latter. J Hand Surg Am 2012; 37:591-6.

3. $\mathrm{Ng} \mathrm{KH}$, Peh WC. Preparing effective illustrations. Part 1: graphs. Singapore Med J 2009; 50:245-9.

4. Ng KH, Peh WC. Preparing effective illustrations. Part 2: photographs, images and diagrams. Singapore Med J 2009; 50:330-4.

Source of support: None

Conflict of interest: None declared

First submitted: $9^{\text {th }}$ March 2018

Published online: $13^{\text {th }}$ March 2018 